\title{
N.I. PLATNICK: Advances in Spider Taxonomy 1992-1995. With Redescriptions 1940-1980.
}

New York Entomological Society, in association with The American Museum of Natural History. 1997, 976 S., ISBN 0-913424-24-2.

Das taxonomische Wissen über Webspinnen ist in einzigartiger Weise aufbereitet. Der ersten Übersicht der Arten und Zitate von taxonomischer Bedeutung von 1758 (1757) bis 1940 bzw. ca. 1954 („Lycosaeformia“, Dionycha, „Cribellata“) von ROEWER (1942, 1955) waren bisher drei Kataloge gefolgt, dank der souveränen Beherrschung und bibliographischen Bewältigung dieses Wissensgebietes durch P.M. Brignoli (L'Aquila, §1986) und N.I. Platnick (New York). BRIGNOLI (1983) fasste zunächst die Neubeschreibungen und Gattungs-Transfers für die Jahre 1940-1980 zusammen, mit einem Addendum für 1979-1982. PLATNICK $(1989,1993)$ hat diese Übersichten für die Perioden 1981-87 bzw. 1988-91 fortgesetzt, unter Berücksichtigung auch der erst nach Roewer erkannten und zunächst unerfasst gebliebenen Synonymien und Transfers bei den schon von Roewer aufgenommenen Arten. Nach nur vier Jahren liegt nun ein weiterer voluminöser Band von nahezu tausend Seiten für den Zeitraum 1992-95 vor!

Enthalten ist zunächst die weltweite Aktualisierung der taxonomischen Information für 106 Familien: Neu- und ergänzende Beschreibungen und Transfers 1992-95; ergänzt durch die taxonomisch bedeutsamen Zitate aus dem Zeitraum 1940-80, die weder einen Transfer noch eine Synonymisierung zur Folge hatten und bisher unberücksichtigt geblieben waren. Darüber hinaus sind in einzelnen Gattungen die als Ergebnis rezenter Revisionen nicht mehr identifizierbaren „Nomina dubia“ aufgelistet, womit man beginnt, den „taxonomischen Ballast“ zu markieren.

Dem neuen Katalog liegen aus dem Berichtszeitraum 913 Arbeiten zugrunde, gegenüber 652 Arbeiten für den Zeitraum 1988-91. Die Bewältigung dieser Informationsflut ist längst auf elektronische Dokumentation angewiesen. Möglicherweise ist dieser Band auch der letzte „gedruckte Katalog“. Die Einleitung deutet die Absicht an, in der Folge die Information der verschiedenen Kataloge zu einer zusammenfassenden „elektronischen“ Datenstruktur zusammenzuführen und zu vereinheitlichen. Jedenfalls hat N. Platnick mit diesem Katalogband den Arachnologen 
wiederum ein wunderbares Instrument beschert: eine weltweite, akribische Übersicht der „neuen“Arten, Nachbeschreibungen, Synonymien, Transfers bei Webspinnen; unter Berücksichtigung auch der schwer zugänglichen bzw. „exotischen" Quellen und der umfangreichen Sammelwerke (z.B. BARRION \& LITSINGER 1995; WUNDERLICH 1995, Beitr. Araneologie 4). Das „äussere Kleid“ ist ansprechend und benutzerfreundlich: Druck, Anordnung des Textes, der Einband. Natürlich ist ein solcher Katalog auch ausserhalb des engen Kreises der taxonomisch interessierten Araneologen von Bedeutung: einmal als Nachschlagewerk für systematische Stellung, Schreibweise und Verbreitung der Taxa, vor allem aber zur Dokumentation von Erforschungsstand und Mannigfaltigkeit.

Die Probleme und Schwierigkeiten in der Bibliographie der Entomologie haben HORN \& SCHENKLING (1929) im Vorwort zum 4. Band ihres ,Index Litteraturae Entomologicae“ in auch heute aktueller und spannender Weise beschrieben, „im Verlauf der Jahrzehnte .. muss der historische Ballast die naturwissenschaftliche Arbeitsmethodik immer mehr zum Erstarren bringen“. Unser aller Dank geht an den Verfasser, dessen neuer Katalog wiederum dazu verhilft, das uferlose Wissen in der Spinnenkunde in kondensierter Form präsent zu erhalten. So ist die Grundlage gegeben, dass taxonomisch/ tiergeographische Forschungen in dieser Ordnung weiterhin Faszination und Anreiz bewahren.

Konrad THALER 